\section{Echinococcus in the orbit: an unusual hideout}

\author{
Hetal Marfatia, Nilam U. Sathe, Subodh \\ Hosagadde, Keya Shah \\ Department of Ear, Nose and Throat, \\ King Edward Memorial Hospital and Seth \\ G.S. Medical College, Mumbai, India
}

\section{Abstract}

Isolated involvement of the orbit by hydatidosis is extremely rare. It is a parasitic infestation due to a tapeworm called Echinococcus Granulosus. It is an uncommon disease in head and neck region as it commonly manifests in the lungs and liver. When seen in head and neck, it has been found in the orbit, paranasal sinuses and salivary glands. Orbital hydatidosis per se forms only $1 \%$ of all types of hydatid disease. It is endemic in Africa, Middle East and Southeast Asian countries including India. In orbital hydatid disease, patients usually present with unilateral proptosis and diminution of vision. Proptosis is classically painless. Total surgical removal remains the mainstay of treatment.

A 30-year-old female belonging to lower socio-economic status, presented with left eye proptosis since 2 years. There was a gradual increase in proptosis associated with diminution of vision over the last 4 months. This finally led to complete loss of vision in a period of two months. Computed tomography scan showed two soft homogenous non-enhancing cystic structures present in the orbit abutting the optic nerve without any intracranial extension; most likely differentials are a developmental cyst, hydatid cyst, epidermoid cyst, rhabdomyosarcoma or a nerve sheath tumor. Magnetic resonance imaging showed two T1 hypointense non-enhancing, T2 hyperintense soft cystic capsulated space-occupying lesions present in the extraconal compartment of orbit abutting the optic nerve, likely to be lymphangioma or a hydatid cyst. Surgery was planned and endoscopic orbital decompression was done. Sago grain-like granules were seen pouring out of the cysts. Cyst walls were completely removed in piecemeal and the specimen was sent for histopathology. Immediate intra-operative reduction of proptosis was seen. The final histopathological report was suggestive of hydatid cyst.

\section{Introduction}

Isolated involvement of the orbit by hydati- dosis is extremely rare. Cystic echinococcosis is a parasitic infestation due to a tapeworm called Echinococcus Granulosus. It is an uncommon disease in the head and neck region as it commonly manifests in the lungs and liver. When seen in the head and neck, it has been found in the orbit, paranasal sinuses, salivary glands. ${ }^{1-4}$ Orbital hydatidosis per se constitutes $1 \%$ of all types of hydatid disease. ${ }^{5}$ It is endemic in Africa, Middle East and Southeast Asian countries including India.

Symptoms generally depend on the site of involvement. In orbital hydatid disease, patients usually present with unilateral proptosis and impairment of vision. Proptosis is classically painless and gradually progressive, which may further lead to restriction of eye movements, exposure keratitis with ocular congestion. In advanced cases there may be papilloedema and complete loss of vision due to optic nerve atrophy. ${ }^{6}$

Relevant literature suggests that a preoperative diagnosis of hydatid is very rarely established. Differential diagnoses like developmental cyst, epidermoid cyst or nerve sheath tumors are considered and the final diagnosis is confirmed only after histopathological examination of the excised cyst. Total surgical removal remains the mainstay of treatment.

\section{Case Report}

A 30-year-old female belonging to lower socio-economic strata, presented with left eye proptosis since 2 years. There was a gradual increase in proptosis associated with diminution of vision over the last 4 months. This finally led to complete loss of vision in a period of two months (Figure 1).

Ophthalmological examination suggested that there was no perception of light in the left eye with restricted ocular movements in all directions. There was associated chemosis and the long-standing proptosis had even led to corneal ulceration, thus creating a hurdle in the assessment of optic disc (Figure 2).

A complete radiological examination was done [ contrast computed tomography (CT) of paranasal sinuses (PNS) and magnetic resonance imaging (MRI) PNS with MRI brain]. CT scan showed two soft homogenous nonenhancing cystic structures present in the orbit abutting the optic nerve without any intracranial extension; most likely differentials being a Developmental cyst, hydatid cyst, epidermoid cyst, rhabdomyosarcoma or a nerve sheath tumor. MRI showed two T1 hypointense non-enhancing, T2 hyperintense soft cystic capsulated space-occupying lesions present in the extraconal compartment of orbit abutting the optic nerve, likely to be lymphangioma or a hydatid cyst (Figures 3 and 4).
Correspondence: Nilam U. Sathe, Department of Ear, Nose and Throat, King Edward Memorial Hospital and Seth G.S. Medical College, Patidar complex 304/ B, Kannamwar Nagar, Vikhroli (East), Mumbai 400083, India.

Tel: +91.02225782801 - Fax: +91.9821309298.

E-mail: drneelam_s@yahoo.co.in

Key words: Echinococcus; Orbit; India; Hydatidosis.

Conflict of interest: the authors declare no potential conflict of interest.

Received for publication: 10 April 2016

Revision received: 31 May 2016.

Accepted for publication: 3 June 2016.

This work is licensed under a Creative Commons Attribution NonCommercial 4.0 License (CC BYNC 4.0).

(C) Copyright H. Marfatia et al., 2016

Licensee PAGEPress, Italy

Surgical Techniques Development 2016; 6:6539 doi:10.4081/std.2016.6539

Surgery was planned and endoscopic orbital decompression was done. Sago grain-like granules were seen pouring out of the cysts (Figure 5). Cyst walls were completely removed in piecemeal and specimen was sent for histopathology. Immediate intra-operative reduction of proptosis was seen.

Final histopathological report was suggestive of hydatid cyst (Figure 6). Post-operatively, proptosis resolved and the ocular movements recovered in all quadrants. However, there was no improvement of vision, possibly because of long standing pressure on the optic nerve.

\section{Discussion}

Isolated intraorbital hydatid cyst is a rare entity and accounts for only $1 \%$ of all hydatidosis in the body (morales). It is caused by a tapeworm of genus Echinococcus. There are three types of species: granulosus, multilocular, vogeli, but the most common is the granulosus variety. Humans are the accidental intermediate hosts, due to exposure to food or water contaminated by feces of definitive host like pigs, sheep, dogs, cattle, etc. Symptomatology depends mainly on the affected organ.

In cases of orbital involvement, painless, progressive unilateral proptosis is usually observed. There may be progressive visual impairment and restriction of eye movements, depending on the size and compression of the optic nerve. Prolonged proptosis may cause secondary complications like ulcerative kerati- 
tis, chemosis of conjunctiva, lid edema. ${ }^{5,7}$ In our case patient had a similar presentation. Children and young adults are commonly affected. Any gender preference is not observed, but left eye is seen to be mostly affected. ${ }^{8}$ In intraorbital lesions, superomedial or superolateral compartment is said to be involved more commonly. In our case it was a superomedial lesion.

Tissue diagnosis by fine needle aspiration cytology (FNAC) is not advised, as it may cause rupture of the cyst, causing acute anaphylaxis and spread. But there are a few reports of FNAC without any complications. ${ }^{9}$ Imaging techniques like ultrasonography (USG), CT and MRI generally give the diagnosis. A welldefined thin walled cyst with a loculated cystic appearance is suggestive of a hydatid cyst. It may be multiloculated or uniloculated. CT and MRI show the presence of daughter cysts and internal septae and also its relation to the optic nerve, intraocular muscles, extent of the disease and bony erosions. ${ }^{10,11} \mathrm{~A}$ chest $\mathrm{X}$ ray and abdominal USG is always advisable to rule out involvement of other organs. Although clinical examination and investigations help, definitive diagnosis can only be made on histopathological examination and hence is considered as the gold standard. Various surgical approaches have been used like transmaxillary or lateral rhinotomy or transconjunctival. In our case endoscopic approach was chosen, considering the patient's age and location of the cyst in the orbit. Uncinotomy was done. Anterior and posterior ethmoidal cells were cleared. Lamina papyracea was removed. The tense extraconal cyst was incised. The cyst collapsed pouring out a lot of fluid and the opening of cyst wall was identified. The interior of the cyst had multiple sago grain-like appearance. The cyst was dissected all around and complete cyst wall was removed piecemeal. Another hidden small daughter cyst was found then, which was removed in a similar manner.

Total surgical excision is the mainstay of treatment as recurrences are quite common. In case of rupture of the cyst during surgery, every attempt should be made for complete removal and thorough hypertonic saline washes should also be given. Albendazole therapy may also be effective in preventing recurrences, ${ }^{12}$ which was given to our patient post operatively.

\section{Conclusions}

Hydatid cyst is a very rare entity in head and neck and is found even more rarely in the intraorbital region presenting as unilateral proptosis. Total surgical excision is the treatment of choice. Only the histopathological report is conclusive and is considered the diagnostic gold standard.

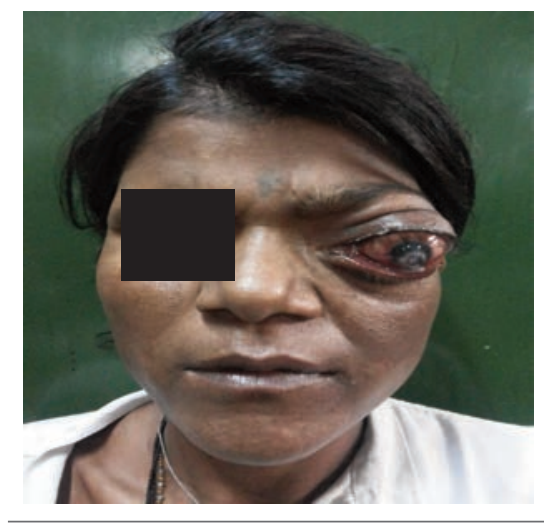

Figure 1. Left proptosis with down and out displacement suggesting superomedial lesion.

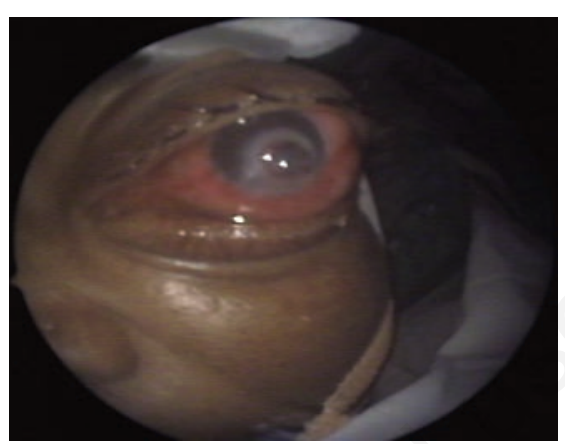

Figure 2. Conjunctival chemosis andcorneal ulceration.

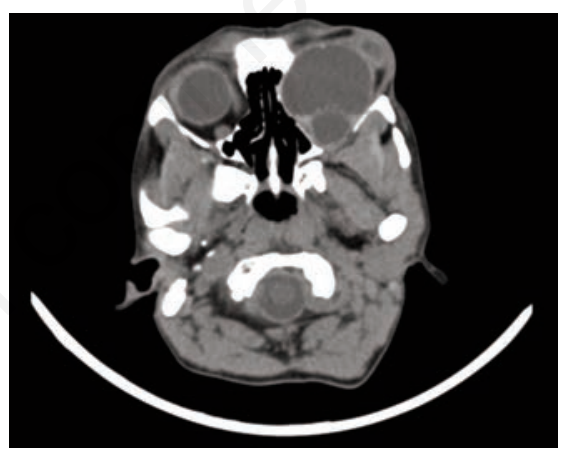

Figure 3. Computed tomography axialview: superomedial location of the two cysts.

\section{References}

1. Benazzou S, Arkha Y, Derraz S, et al. Orbital hydatid cyst: review of 10 cases. J Craniomaxillofac Surg 2010;38:274-8.

2. Cooney RM, Flanagan KP, Zehyle E. Review of surgical management of cystic hydatid

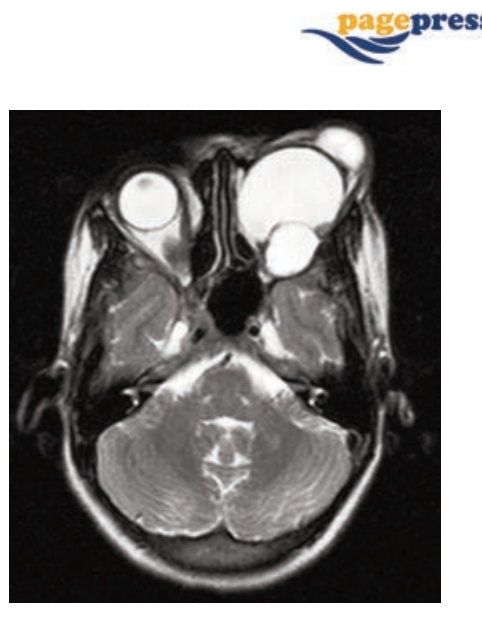

Figure 4. T2-magnetic resonance imaging: hyperintense lesions suggestive of fluid within cysts.

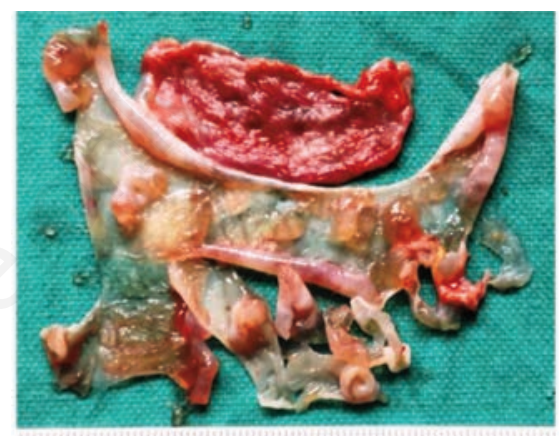

Figure 5. Sago grain-like granules pouring out of the cysts.

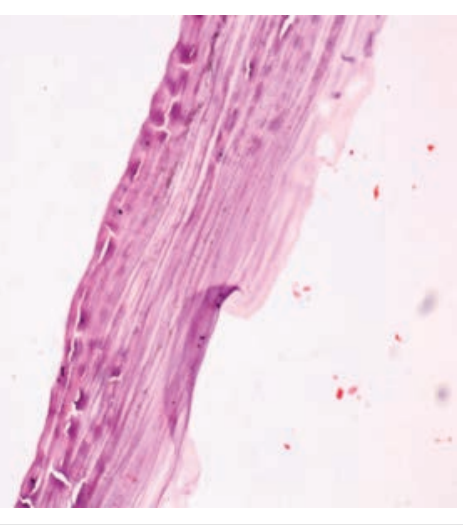

Figure 6. Final histopathological reportsuggests hydatid cyst.

disease in a resourse limited setting: Turkana, Kenya. Eur J Gastroenterol Hepatol 2004;16:1233-6.

3. Megalamani SB, Balasubramanium D, Gopalakrishnan S, Saxena SK. Hydatid cyst of the maxillary sinus. Clin Rhinol Int J 2011;4:107-9.

4. Fradis M, Podoshin L, Golstein Y, et al. 
Cervical echinococcal hydatid cyst. J Laryngol Otol 1989;103:435-7.

5. Morales AG, Oscar-Croxatto JO, Crovetto L, Ebner R. Hydatid cysts of the orbit: a review of 35 cases. Ophthalmology 1988:95:1027-32.

6. Bamashmus MA, Al-Shabooti AA. Orbital hydatid cyst: an unusual presentation. Saudi J Ophthal 2006;20:146-8.

7. Sami A, Achori M, Harouch M, et al. Intra- orbital hydatid cyst. 10 cases. Neurochirurgie 1995;4:398-402.

8. Limaiem F, Bellil S, Bellil K, et al. Primary orbital hydatid cyst in an elderly patient. Surg Infect (Larchmt) 2010;11:393-5.

9. Berkiten G, Topaloglu I. Submandibular hydatid cyst fistulized into the oral cavity. B-ENT 2013;9:251-3.

10. Benhammou A, Benbouzid MA, Bencheikh $\mathrm{R}$, et al. Hydatid cyst of the neck. B-ENT
2007;3:201-3.

11. WHO Informal Working Group. International classification of ultrasound images in cystic echinococcosis for application in clinical and field epidemiological settings. Acta Trop 2003;85:253-61.

12. Katilmi H, Oztürkcan S, Ozdemir I, et al. Primary hydatid cyst of the neck. Am J Otolaryngol 2007;28:205-7. 